\title{
La restauración absolutista de 1814 en los rota- tivos estadounidenses. Imagen de una decepción
}

\author{
Rebeca Viguera RuIZ \\ Universidad de La Rioja ${ }^{1 *}$ \\ rebeca.viguera@unirioja.es
}

Recibido: 02/02/2014

Aceptado: 05/09/2014

\section{RESUMEN}

Este trabajo trata de presentar el modo en que la prensa estadounidense retransmitió los cambios sociopolíticos de 1814 en España, en un tono de crítica y decepción que fue reflejo de la opinión pública generada en Estados Unidos al respecto. Con tal fin se ha buscado plasmar la versión oficial de los papeles y autoridades españolas que llegó a los editores norteamericanos, junto con la visión crítica que éstos ofrecieron como consecuencia de una lectura detallada y reflexiva de los primeros. Una imagen de condena y decepción diferente a la que en 1812 se había esbozado en esa misma prensa sobre la Constitución de Cádiz.

Palabras clave: Absolutismo, Legitimismo, Fernando VII, Prensa, Estados Unidos, Constitución, Libertades

\section{The restauration of absolutism in 1814 in American newspapers}

\begin{abstract}
This paper seeks to present the way in which the Northamerican Press communicated the socio-political changes occurred in Spain in 1814: reflecting the high level of criticism and disappointment generated among the people of the United States. To this end, the official version of the Spanish papers and authorities revealed to the US publishers will be considered together with the criticism they offered as a result of further considerations about it. It was an image of disappointment the one presented in the Press, and it was a different one from the one outlined in 1812 about the Constitution of Cádiz.
\end{abstract}

Key words: Absolutism, Legitimism, Ferdinand 7th, Press, United States, Constitution, Freedom \& Liberty

\footnotetext{
$1 *$ Este trabajo se enmarca dentro del Proyecto Nacional de Investigación «Retórica e Historia. Los discursos parlamentarios de Salustiano de Olózaga (1847-1871)», Ref. FFI2011-23519/FILO del Ministerio de Economía y Competitividad, e investigador principal D. José Antonio Caballero López. Dejo constancia de mi agradecimiento a esta institución.
} 
The constitution of England, this may be so; the «legitimate King» also took the first moment of power to annul that constitution; and the same thing has happened in

Spain. In both countries the seed of new revolutions is laid; and tyranny, instead of the «freedom» they hoped for in the «restoration» of their Kings, may give it ripeness in a very little time. Nothing but a keen recollection of late sufferings will prevent new thrones and convulsions. The people have had a gleam of liberty, and the time now is to secure it, or lose it for ages. Columbian Patriot (Middlebury, VA), 14 de septiembre de 1814, p. $1^{2}$

En un momento en el que parecían estar triunfando en Europa las tan ansiadas y valoradas premisas de freedom y liberty para los recién creados Estados Unidos de América, los Spanish Affairs que tuvieron lugar en los primeros meses de 1814 imprimieron un rumbo radicalmente opuesto a los acontecimientos y abrieron el camino a los valores monárquicos que el Congreso de Viena habría de sancionar definitivamente en 1815, dando origen a lo que la historiografía conoce como Europa de la Restauración.

En 1812 la prensa estadounidense había emitido críticas muy positivas en torno a la Constitución de Cádiz y los valores liberales que en España se habían establecido como consecuencia de su aprobación. Se favorecía con ella la participación de toda una nueva clase política en las decisiones de las Cortes y el Gobierno, se daba al pueblo un poder público hasta entonces desconocido y suponía la culminación de una lucha constante y sacrificada que se mantenía en el país desde 1808 frente al ejército imperial de Napoleón Bonaparte.

A principios de 1814 Fernando VII regresó de Francia, acabó con toda la obra liberal de las Cortes de Cádiz y decretó el absolutismo como forma de gobierno legítima. Viraje político respaldado por las potencias Europeas con mayor fuerza en el Congreso de Viena pero condenado por otros poderes internacionales - como Estados Unidos- que habían iniciado ya el camino hacia sistemas liberales de cariz democrático. Dentro de estos últimos, la opinión pública estadounidense ofrecería un claro rechazo a esa «restoration of legitimate kings» que mencionaba el texto de inicio ${ }^{3}$. Tal como los periódicos Columbian Patriot o Albany Argus reflexionaban en esos momentos, en manos del pueblo español quedaba entonces la decisión de mantener y asegurar su libertad o de perderla durante años.

En estas páginas se intentará analizar el modo en que la prensa estadounidense retransmitió a lo largo del año 1814 los cambios socio-políticos acaecidos en la realidad española, así como el tono de crítica y decepción que puede apreciarse en la opinión pública reflejada en dichas páginas de Estados Unidos. Ello implica tener en cuenta -muy sucintamente- dos aspectos relacionados: por un lado la propia figura de Fernando VII como monarca y su implicación con el Antiguo Régimen; por otro, el hecho concreto de la derogación de la Constitución de 1812 y su repercusión mediática. Ambos analizados a su vez desde dos perspectivas complementarias: la versión

\footnotetext{
2 La misma referencia también en Albany Argus (Albany, NY), 2 de septiembre de 1814, p. 2.

3 Se ha decidido mantener todas las citas textuales en inglés con el fin de no alterar en la traducción su sentido original, y poder comprobar las palabras exactas que se utilizan en las diferentes expresiones que se han incorporado en estas páginas.
} 
oficial de los papeles y autoridades españolas que llegó a los editores norteamericanos, y la visión crítica que éstos ofrecieron como consecuencia de una lectura detallada y reflexiva de los primeros ${ }^{4}$. Sin duda una imagen de condena y decepción muy diferente a la que dos años antes se había esbozado en esa misma prensa en reflejo de un país leal que había iniciado su andadura constitucional.

\section{El momento histórico en torno a 1814. Un apunte}

De sobra es conocido que entre 1808 y 1814 se sucedieron en España dos procesos históricos de gran impacto para la evolución política, social e ideológica del país durante el resto de la centuria. Por un lado la invasión napoleónica y la consiguiente lucha constante de los españoles frente a la ocupación francesa. Y de otro, la convocatoria de unas Cortes que conllevó la génesis y proclamación de la primera constitución liberal de la Nación en 1812.

El conflicto armado contra Francia iniciado en el año 1808 y la salida del país de la familia real española, había llevado al mismo a una situación de vacío de poder que estuvo en la base de la proliferación de movimientos Junteros por todo el país, la convocatoria de unas Cortes Extraordinarias en 1810 con el fin de encontrar solución a la situación general de crisis del país, y la aprobación de la Constitución Politica de la Monarquía Española en Cádiz en 1812. En esos momentos tuvo lugar igualmente el surgimiento de nuevos espacios de actuación política entre los que cobraron importancia los municipios y las capitales de provincia como fuentes de soberanía. Pese a todos los avances políticos y económicos que se desarrollaron en esos años, los diferentes gobiernos no fueron capaces de dar respuesta a la acuciante situación hacendística ni a la reorganización socio-política de la estructura estamental previa. Estando así las cosas en el país, en diciembre de 1813 Napoleón reconoció a Fernando VII como monarca legítimo del trono español a través del tratado de Valençay. En el mes de marzo de 1814 el rey cruzó la frontera por Cataluña y se dirigió hacia Valencia, con un apoyo popular generalizado que mostraba el deseo de los españoles de que se restableciera el orden legítimo en el trono que había sido usurpado en 1808 por Bonaparte. El 4 de mayo de 1814 Fernando VII decretaría de manera oficial el restablecimiento de su marca de gobierno absolutista declarando nula la obra de las

4 Es preciso señalar que no se ha podido detectar la autoría de la mayor parte de los artículos de opinión o editoriales que se han utilizado como referencia en el presente trabajo. Prácticamente la totalidad de las noticias aludidas proceden de los espacios dedicados en los periódicos a los telegramas o noticias internacionales que, como se especifica más adelante, proceden generalmente de recortes de prensa europeos-, y carecen de autor reconocido en las páginas de referencia. Cabe pensar que aquellas que fueron presentadas como textos de opinión proceden de la editorial del periódico y dependían de la línea ideológica de los mismos. Aunque es igualmente necesario matizar que, independientemente del posicionamiento ideológico de cada uno de ellos, no se han detectado diferencias extremas o radicalmente opuestas en el tratamiento y caracterización que se hace de la decisión monárquica de Fernando VII de la derogación de la Constitución de 1812: siempre negativa desde el punto de vista del avance político del país tal como se deduce de las citas presentadas. Lo que por supuesto no significa que los enfrentamientos político-ideológicos entre los papeles federales y los demócrata-republicanos en materia de política interior y exterior no fueran una constante; tema que por su dimensión podría ser objeto de un interesantísimo estudio más amplio y específico que el presente que tuviera por objeto el análisis del impacto de los sucesos europeos del momento en la política nacional estadounidense. 
Cortes de Cádiz y su labor legislativa. Desde ese momento, y hasta 1820, se asistiría a una vuelta al orden tradicional respaldado igualmente por la Europa de la Restauración y del Congreso de Viena.

Es esas mismas fechas los jóvenes Estados Unidos de América seguían luchando por consolidar y mantener la independencia obtenida unas décadas antes frente a Gran Bretaña. En torno a 1812, junto con debates económicos y sociales de importancia para la política interna del país, la preocupación principal de la nueva nación era la coyuntura comercial y diplomática exterior. James Madison había sucedido en la presidencia del país a Jefferson, ambos del partido republicano, y el 18 de junio de 1812 se vio obligado a declarar la guerra a Gran Bretaña e Irlanda tras la reunión mantenida por el duodécimo congreso estadounidense que había dado comienzo en el mes de noviembre del año anterior ${ }^{5}$. La situación de bloqueo comercial generada por la propia realidad que se vivía en Europa en el marco de las guerras napoleónicas provocó numerosas pérdidas para el comercio y los intereses de Estados Unidos ${ }^{6}$. Éstos observaron cómo sus derechos de comerciar libremente con otras naciones se vieron vulnerados por Gran Bretaña en un intento de esta última de poner freno tanto a la expansión de Francia como al crecimiento progresivo de sus antiguas colonias del Atlántico Norte. Fue así como surgió aquella War of 1812, también conocida como Segunda Guerra contra Inglaterra, Guerra Anglo-Americana de 1812 o Segunda Guerra de la Independencia estadounidense ${ }^{7}$. Por el propio impacto que todo ello tenía en la economía, la política y la moral estadounidenses, las noticias relacionadas con estas cuestiones fueron las más frecuentemente señaladas en sus periódicos en relación con la Europa del momento. Y en la medida en que dicho enfrentamiento se prolongó hasta principios de 1815, todavía en 1814 seguía siendo un tema de prioridad.

Por tanto, teniendo presente la realidad de ese momento histórico tanto en España como en Estados Unidos, aunque brevemente apuntada, se observa en ella la forja de los primeros fundamentos liberales de la política española, pero también el intento de los recién nacidos Estados Unidos de delimitar su nuevo estatus político, de perfilar

5 NEVINS, Allan: American Press opinion. Washington to Coolidge. A documentary record of editorial leadership and criticism, 1785-1927. United States: D.C. Heath and Company, 1928, p. 4.

6 «The vessels and cargoes of American merchants were seized and confiscated under various decrees, founded in different pretexts, none of which had then the semblance of right to support them», en Republican Star (Easton, MD), 2 de junio de 1812, p. 1. Sobre este conflicto son innumerables las referencias que podrían citarse. Entre ellas, como apunte de su importancia para la historia posterior del país, pueden consultarse las de Borneman, Walter R.: 1812: the War that Forged a Nation, New York: Harper Perennial, 2004; Remini, Robert V.: The Battle of New Orleans: Andrew Jackson and America's First Military Victory. Chicago: Penguin Group, 2001; Gilpin, Alec R.: The War of 1812 in the Old Northwest. East Lansing: Michigan State University Press, 2012; Stagg, J. C. A.: The War of 1812: conflict for a continent. New York: Cambridge University Press, 2012; Philpott, Don: The U.S. Navy pictorial history of the War of 1812. Lanham, Md.: Rowman \& Littlefield, 2012; Roosevelt, Theodore: The naval War of 1812. New York: Modern Library, 1999; o algunas referencias coetáneas como puede ser la recopilación de Armstrong, John: Notices of the War of 1812. New-York: George Dearborn, publisher, 1836 [-1840].

7 Un enfrentamiento que fue concebido como «impolitic, unjust and ruinous to the present peace», así como «destructive of our freedom, our constitution, our powers of selfgovernment, and our political existence»; pero a la vez inevitable para lograr la seguridad económica del país. Una guerra inevitable que ocupó la mayor parte de reflexiones políticas internacionales en las páginas de la prensa norteamericana del momento. Referencias en Weekly Messenger, 24 de julio de 1812, p. 4. 
la conciencia nacional y de consolidar su independencia y sus intereses económicos en el panorama internacional.

Es en ese escenario donde debe entenderse el contenido de las noticias que sobre España se transmitían en los periódicos estadounidenses. Sin duda un momento convulso en ambas orillas del Atlántico norte donde las grandes potencias occidentales luchaban aún por superar parámetros políticos imperialistas y absolutos a favor de nuevos sistemas de gobierno liberales y, en algunos casos, democráticos ${ }^{8}$.

\section{La prensa norteamericana en 1814: breves notas explicativas}

Sin perder de vista el hecho de que, de manera general, «the press is a pivotal force in society, indispensable for producing social cohesion, asserting cultural identity, and upholding the principles of democracy» ${ }^{9}$, es necesario entender que en Estados Unidos la prensa jugó un papel esencial en la configuración de la nación y de la conciencia social estadounidense. Toda la historiografía norteamericana, coetánea y más reciente, coincide en afirmar que «the press played a significant role in founding the new republic and in its subsequent development. [...] As the parties developed and grew more powerful, so did the newspapers ${ }^{10}$.

Desde prácticamente sus orígenes los actores políticos y las instituciones han confiado en ella como medio de difusión de información al público, y el público ha confiado en los medios de comunicación como medio de obtener información sobre los procesos políticos y el trabajo de las instituciones. Para las fechas de estudio de este texto, «in the early days of the republic, the American press was typically financially dependent on the resources of political leaders who provided patronage to editors and publishers, start-up loans for newspapers, and continued funds for operation -as well as often, guaranteed readerships and news material itself $\rangle\rangle^{11}$. Tras la independencia se erigió como una verdadera herramienta de movilización social, un medio de generar opinión afín a unos principios morales y políticos determinados, y «a major force

8 En una casuística muy compleja de tendencias y posicionamientos políticos -difícilmente identificables con etiquetas sencillas-, que conjugaban en ocasiones el pasado y el presente, los términos políticos tradicionales con la nueva carga semántica de los conceptos modernos en la conformación general de los estados occidentales contemporáneos. Vide por ejemplo, entre otras referencias, algunas consideraciones al respecto en las diferentes entradas de: FERNÁNDEZ SEBASTIÁN, Javier: Diccionario politico y social del mundo iberoamericano. La era de las revoluciones, 1750-1850. Madrid: Centro de Estudios Políticos y Constitucionales, 2009.

9 BRENNEN, Bonnie \& HARDT, Hanno: The American Journalism. History reader. Critical and primary texts. London: Routledge, Taylor \& Francis, 2011, p. 1.

10 KOBRE, Sidney: Foundations of American Journalism. Westport, Connecticut: Greenwood Press, 1970, p. V. Este mismo autor señaló, al hilo de esta cuestión que «American press developed from the small, weak sheets of the colonial days to the large, powerful metropolitan newspapers of the Civil war period. Eighteenth and Nineteenth Century journalism provided the foundation stones of the modern Twentieth Century newspaper, magazine, radio and television», Ibid.

11 DAVIS, Richard: The Press and American politics. The new mediator. New Jersey: Prentice Hall, 2001, p. 7. Pese a la relación que todavía después tuvo y ha mantenido con los poderes políticos, la prensa dejaría a finales del XIX de depender del gobierno o de individuos particulares para lograr la estabilidad económica o financiación necesaria. 
in bringing debate out of the exclusive hands of the elite, and into a wider public forum $\rangle^{12}$. Precisamente por ese motivo el contenido de los periódicos sería fundamentalmente de interés político o económico nacional. Ello sumado al hecho de que los ejemplares de la generación posterior a la Revolución y a la independencia «were small, dingy, once-folded sheets which printed fragmentary commercial and political intelligence, some advertisements and long editorials which usually resembled daily instalments of a pamphlet, or were actually such instalments. If written by the editorial himself, they might unreservedly be called editorials» ${ }^{13}$. Es ésta y no otra la prensa que ha de analizarse para comprobar la visión que se reflejó en ella de la España del momento. Un tipo de prensa que estaba empezando a expandirse tanto desde una perspectiva formal como ideológica, y que tenía numerosas limitaciones derivadas de su más o menos reciente desarrollo y las dificultades en su distribución.

Hay que tener en cuenta que durante las décadas finales del siglo XVIII y a comienzos del XIX los periódicos norteamericanos dependían de las noticias que se transportaban por correo ordinario de un lugar a otro del territorio, «mails carried typically by foot or on horseback, often along Indians trails» $\mathrm{y}$ cuyas «limitations became all too apparent» ${ }^{14}$. A la altura de 1814:

Large parts of the South remained without postal service. Roads were concentrated along the Atlantic coast. Settlers in the western Carolinas, and virtually all of Georgia and Alabama, had to rely on traveller for their infrequent communication with the rest of the country. The South Atlantic Coast and southern Mississippi Valley remained isolated from one another -indeed, much of the communication between these two centers of southern civilization flowed through North-eastern cities, especially New York. The most important postal development involving vehicles was the growing use of stagecoaches, necessitated in part by the mass of newspaper mails.

Even where stagecoach service was firmly established, problems still plagued newspaper delivery. When roads became muddy, driers sometimes left the heavy newspaper portmanteaus behind to lighten the load. Carrying the newspaper bags on the dashboard, exposed to the weather and the drivers' feet, provoked complaints from subscribers whose papers were damaged or destroyed ${ }^{15}$.

Las dificultades derivadas de este lento transporte son importantes para comprender la naturaleza y contenido de los periódicos estadounidenses en torno a 1814 . El flujo de información por tierra era complejo, y el marítimo suponía altos costes económicos a la vez que seguía siendo lento una vez alcanzaba los puertos e iniciaba su redistribución terrestre. Ello provocó que el intercambio de noticias entre las publicaciones de los diferentes estados fuera la base de la información contenida en sus

12 MELLEN, Roger P.: The origins of a free Press in prerrevolutionary Virginia. Creating a culture of political dissent. New York: The Edwin Mellen Press, 2009, p. 93.

13 NEVINS, Allan: American Press opinion..., op. cit., p. 4.

14 KIELBOWICZ, Richard B.: News in the mail. The Press, Post Office and Public Information, 17001860s. Connecticut: Greenwood Press, 1989, p. 46.

15 Ibid. p. 47. Para más datos pueden consultarse los trabajos de RICH, Wesley Everett: The History of the United States Post Office to the year 1829. Lawrence, Mass.: Quarterman Publications, 1977 y HOLMES, Oliver W. \& ROHRBACH, Peter T.: Stagecoach East. Stagecoach days in the East from the Colonial period to the Civil War. Washington, D.C.: Smithsonian Institution Press, 1983. 
páginas, junto con un número creciente de anuncios y debates internos de los editores del periódico de acuerdo a los debates políticos del Congreso en cada momento.

«The proceedings, laws, and debates of various legislative bodies, political and miscellaneous essays, the letters to the editors, commercial notices, and advertising constituted the bulk of the paper», y en medio de esta amalgama de contenidos, los editores del ochocientos «were enthusiastically dedicated to publishing the best exchanges and lamented the poverty of their columns when the mails failed to bring timely news» ${ }^{16}$. Estas eran las bases que permitían sacar adelante las diferentes tiradas diarias o semanales de cada territorio. Escasas tiradas con noticias internacionales que tuvieran que ver con sus relaciones comerciales o bases de política interior y basadas en una información atrasada que se repetían de manera permanente en las mismas fechas en los diferentes periódicos de cada Estado.

A pesar de que, por todos esos motivos, la comunicación diaria seguía siendo fundamentalmente oral entre los norteamericanos, los periódicos se habían convertido sin lugar a dudas en elementos indispensables de la actividad política entre $1800 \mathrm{y}$ $1815^{17}$. Tras la guerra de 1812 su número se incrementó de manera pausada como consecuencia del conflicto y sus consecuencias, mientras que a partir de la década de 1820 tuvo lugar «a dramatic increase in newspaper delivery», «the greatest relative increase in spending on newspaper postage» en la zona media del Atlántico ${ }^{18}$. Sin poder detenernos aquí en esa evolución y desarrollo de la prensa estadounidense como vía de expresión social y herramienta de actuación política, ya aludida, la realidad es que a principios del siglo XIX acercó el mundo exterior a la población ${ }^{19}$.

La difusión de las noticias internacionales dependía en último término del sistema postal heredado de las antiguas colonias, pero a su vez su propia recepción se producía con dos o tres meses de retraso respecto a las fechas en que se producía. «European news took two or three months to reach western papers, and even dispatches from the East were more than a month old. Nevertheless, when they did arrive, the news absorbed more than two-thirds of the available space in the newspapers. Revenue from advertisements was small for years» ${ }^{20}$.

Lejos de la velocidad actual que ofrecen las nuevas tecnologías en la transmisión de la información, los editores y propietarios de aquellos periódicos dependían fundamentalmente de cartas de individuos particulares, de los documentos o testimonios que transportaban los barcos provenientes de Europa y de los periódicos que estos mismos llevaban, así como de los intercambios de papeles, informes o documentos que se hacían entre las propias colonias ${ }^{21}$.

16 OSTHAUS, Carl R.: Partisans of the Southern Press. Lexington, Kentucky: The University Press of Kentucky, 1994, p. 4.

17 FORMISANO, Ronald P.: The Transformation of Political Culture: Massachusetts' parties, 1790s-1840s. New York: Oxford University Press, 1983, p. 175.

$18 \mathrm{Y}$ «between the close of the War of 1812 and the advent of the telegraph saw the circulation of news speeded up throughout the nation». KIELBOWICZ, Richard B., News in the mail..., op. cit., pp. 71, 73 y 74.

19 Ibid. p. 5.

20 KOBRE, Sidney, Foundations of American Journalism ..., op. cit., p. 205.

21 Sobre estas cuestiones son de utilidad las reflexiones del ya clásico trabajo de KOBRE, Sidney: Development of American Journalism. Iowa: WM.C.Brown Company Publishers, 1969, pp. 23 y ss. 
Para el caso concreto de España, aquellas referencias llegaban a través de los papeles y publicaciones de La Coruña y Cádiz (dos de los puertos de salida más importantes del país hacia América), que reflejaban el contenido de otros periódicos españoles o resúmenes de estos mismos, así como de otras publicaciones o noticias recogidas y publicadas en otros países europeos, Londres y París fundamentalmente. Por supuesto había otras fuentes, pero esas eran las más comunes, de la mano de la información individual que particulares, soldados o comerciantes transmitían en sus viajes, la mayor parte de ellos vía previa por Cuba, y que se reflejaban en los periódicos en la sección de correspondencia. No debe obviarse que a principios del ochocientos «the most interesting reading, even in some of the most important papers, was found in the letters» ${ }^{22}$.

\section{La figura del monarca español en los periódicos estadounidenses}

De la mano de aquellas Letters, la mayor parte de las referencias a la situación europea y española a principios del siglo XIX apareció bajo titulares genéricos como Spanish Affairs, Foreign affairs from Spain, Foreign News, Highly interesting o European News, entre otros. Tras esas mismas cabeceras apareció igualmente la información referente al retorno de Fernando VII como monarca legítimo del trono español, a su restauración y a su obra posterior. Se trató de reseñas y datos que fueron compartidos por la mayor parte de los periódicos tras su recepción desde España u otros puntos de Europa, por lo que su trascripción, traducción o interpretación fue muchas veces idéntica o muy similar ${ }^{23}$. Y a su vez referencias que complementan la visión de la derogación de la Constitución con un retrato de quien la ejecutó.

Por un lado se verá a continuación la información oficial contenida en los documentos, cartas o periódicos públicos procedentes de la Península que retransmitía el reflejo de lo que el propio monarca y las nuevas autoridades decretaban y hacían público, junto con otras percepciones sociales más subjetivas sobre el sentimiento real del pueblo ante el giro de los acontecimientos tras 1814. Así podrá observarse cómo las líneas que retransmitieron la versión oficial llegada desde España en los papeles públicos mostraban «great joy and enthusiasm on the restoration of their legitimate

22 LEE, James Melvin: History of American Journalism. New York: Garden City Publishing Co., 1923, p. 145. Aunque no se profundiza en esta cuestión, puede tenerse en cuenta el hecho de que «after the Constitution was adopted political leaders found that they needed mouthpieces for a wider expression of their views. They divided themselves into parties of which the common people knew little or nothing. To get the people to take sides on political questions they founded newspapers which, while giving a little news, [...] for politics tended to make the journals of the period not newspapers in the modern sense of the term, but chiefly political tracts». Ibid. p. 101 .

23 Los mismos periódicos reconocen este intercambio de noticias y los préstamos entre unos y otros al inicio de los artículos vinculados a los asuntos exteriores e información internacional. En New Jersey Journal (Elizabethtown, NJ), 19 de abril de 1814, p. 1, se pone de manifiesto que las noticias de Europa habían llegado «from the Boston Centinel, April 6». Otro ejemplo aparece en ese mismo diario, el 11 de octubre de 1814 donde se traduce un comunicado emitido por Fernando VII tomado del Suplemento de la Gazeta de Madrid y que había sido recogido en Boston (Saturday Evening), el 1 de octubre; plasmado posteriormente en New York Gazette, el 4 de octubre. 
Sovereign» ${ }^{24}$; un claro deseo general de que el rey volviera a ocupar el trono que le correspondía y terminara de expulsar a los que se entendían como poderes usurpadores que desde Francia se habían instalado en el país tras 1808. Alegría y tranquilidad iniciales motivadas por la promesa de la convocatoria inmediata de nuevas cortes y el establecimiento de una nueva constitución ${ }^{25}$, y que no se correspondían en realidad con el sentimiento de otros sectores más exaltados de la población, tanto españoles como extranjeros residentes en el país en ese momento, que habían apostado por el cambio de régimen político tras 1812 .

\subsection{El reflejo de una realidad internacional en la prensa de Estados Unidos}

En el marco histórico y periodístico aludido, las primeras noticias que llegaron a los periódicos norteamericanos en relación con el proceso que restauró la monarquía absolutista en España, tienen que ver con los tratados y relaciones diplomáticas mantenidas entre esta última y Francia. Negociaciones que darían como resultado la liberación de Fernando VII por parte de Napoleón, el regreso del primero al trono español y un acuerdo de paz que implicaba la recuperación de los intereses territoriales y comerciales de ambos países previos a la guerra. Coincidiendo en el tiempo, se dio igualmente noticia de los rumores sobre la inminente entrada del monarca en el país y de la decisión de las Cortes de no legitimar a aquél a menos que reconociera la Constitución de 1812 y prestara juramento a la misma ${ }^{26}$.

Así, en las páginas de prácticamente todos estos periódicos puede leerse la trascripción, traducción o resumen de los tratados o decretos resultados de dichos pactos; con especial atención a la paz entre el Emperador y El Deseado que permitiría a este último regresar con un poder absoluto a España y recuperar el control sobre la política del país. Pese a que las negociaciones se habían iniciado en noviembre de 1813 y se concluyeron en Valençay el 11 de diciembre del mismo año, no se conoció de manera generalizada en España hasta el mes de febrero de 1814. Como consecuencia de ello, la noticia no aparecería reflejada de manera reiterada en Estados Unidos hasta el mes de abril de ese $a_{n}{ }^{27}$, procedente fundamentalmente de Madrid, Cádiz o La Coruña y

24 Se observa en New Hampshire Sentinel (Keene, NH), 18 de junio de 1814, p. 3; Federal Republican (Baltimore, MD), 9 de agosto de 1814, p. 3; Daily National Intelligencer (Washington, DC), 28 de julio y 9 de agosto de 1814, p. 3 y p. 3; New York Gazette (New York, NY), 1 de agosto de 1814, p. 3.

25 Así reconocido en Farmer's Cabinet (Amherst, NH), 31 de octubre de 1814, p. 3; Gleaner (Wilkesbarre, PA), 11 de noviembre de 1814, p. 3 o Rhode Island (Newport, RI), 28 de octubre de 1814, p. 3.

26 Sun (Dover, NH), 9 de abril de 1814, pp. 1-2; Connecticut Journal (New Haven, CT), 11 de abril de 1814, p. 2; Columbian Register (New Haven, CT), 3 de mayo de 1814, p. 4; American and Commercial Daily Advertiser (Baltimore, MD), 11 de abril de 1814, p. 2; Baltimore Patriot (Baltimore, MD), 10 de abril de 1814 , p. 2; Hallowell Gazette (Hallowell, Me), 13 de abril de 1814, p. 2; Constitutionalist (Exeter, NH), 12 de abril de 1814, p. 2; Dedham Gazette (Dedham, MA), 8 de abril de 1814, p. 2; New England Palladium (Boston, MA), 5 de abril de 1814, p. 2; Repertory (Boston, MA), 7 de abril de 1814, p. 1; Ohio Register (Clinton, OH), 17 de mayo de 1814, p. 114, Newport Mercury (Newport, RI), 8 de abril de 1814, p. 2; Enquirer (Richmond, VA), 13 de abril de 1814, p. 2; Virginia Patriot (Richmond, VA), 16 de abril de 1814, p. 2; Frankfor Argus (Frankfort, KY), 30 de abril de 1814, p. 3, en Republican Farmer (Bridgeport, CT), 13 de abril de 1814, p. 2 o Connecticut Journal (New Haven, CT), 11 de abril de 1814, p. 2.

27 Noticia de ello se da, por ejemplo, en New Jersey Journal (Elizabethtown, NJ), 19 de abril de 1814, p. 3 «confirmed by the Spanish papers» de 3 de febrero. Igualmente aparece en ese mismo diario en la p. $1 \mathrm{o}$ 
fruto de la demora en la transmisión de noticias ya comentada. Primero se transmitió el desarrollo de las reuniones ${ }^{28}$, e inmediatamente después la sanción del texto oficial con las firmas del Conde de Laforest, comisionado de Napoleón, y H. Wellesley, Vizconde de Castelereagh y embajador plenipotenciario de Fernando VII ${ }^{29}$.

Al documento oficial acompañaron numerosas referencias al proceso que rodeó su firma, los encuentros diplomáticos o incluso las intrigas del Emperador francés para evitar el regreso del monarca español ${ }^{30}$. Testimonios en los que se ponía de manifiesto la animadversión contra Napoleón que estaba latente en el territorio norteamericano $^{31}$, señalando, entre otras cosas, que su «unjust attempt to subjugate Spain, has always been thought one of his most impolitic plans. He could more easily have governed that country in the name of its imbecile Monarch, than by all his armies, at

en Centinel of Freedom (Newark, NJ), 26 de abril de 1814, p. 1; Missouri Gazette and Public Advertiser (St. Louis, MO), 14 de mayo de 1814, p. 3; Star (Raleigh, NC), 18 de marzo, 19 de abril \& 5 de septiembre de 1814, p. 42, p. 67 y p. 122 respectivamente; Constitutionalist (Exeter, NH), 12 y 26 de abril de 1814, pp. 2 y 2; Farmer's Cabinet (Amherst, NH), 11 de abril de 1814, p. 2 y repetido 2 de mayo de 1814, p. 2; Concord Gazette (Concord, NH), 26 de abril de 1814, p. 2; New Hampshire Sentinel (Keene, NH), 30 de abril de 1814 , p. 2; Portsmouth Oracle (Portsmouth, NH), 9 de abril de 1814, p. 2; Sun (Dover, NH), 9 de abril de 1814 , p. 2, Western Citizen (Paris, KY), 30 de abril y 7 de mayo de 1814, p. 1 y p. 2, Connecticut Journal (New Haven, CT), 11 y 25 de abril de 1814, pp. 2-2, Connecticut Herald (New Haven, CT), 12 de abril de 1814, p. 2, Connecticut Mirror (Hartford, CT), 25 de abril de 1814, p. 2, Baltimore Patriot (Baltimore, MD), 29 de marzo y 10 de abril de 1814, pp. 2 y 2; Engine of Liberty and Uniontown Advertiser (Uniontown, MD), 28 de abril de 1814, p. 1 o Hornet (Fredericktown, MD), 27 de abril de 1814, pp. 3-4, Weekly Visiter (Kennebunk, ME), 9 de abril de 1814, p. 2, Hallowell Gazette (Hallowell, ME), 13 de abril de 1814, p. 2, entre otras muchas.

28 En este caso puede comprobarse en «Late Foreing News. Late from France» de Farmer's Cabinet (Amherst, NH), 14 de marzo de 1814, p. 2, o en Norwich Courier (Norwich, CT), 27 de abril de 1814, p. 1, American Mercury (Hartford, CT), 29 de marzo de 1814, p. 3 y Connecticut Spectator, 4 de mayo de 1814, p. 2. El decreto íntegro en Frankfort Argus (Frankfort, KY), 14 de mayo de 1814, p. 1.

29 New Hampshire Gazette (Portsmouth, NH), 14 de junio de 1814, pp. 1-2. Más ejemplos en Farmer's Cabinet (Amherst, NH), 13 de junio de 1814, p. 2; New Hampshire Sentinel (Keene, NH), 18 de junio de 1814, p. 2; Portsmouth Oracle (Portsmouth, NH), 11 de junio de 1814, p. 2; American Watchman (Wilmington, DE), 11 de junio de 1814, p. 2; Delaware Gazette and State Journal, (Wilmington DE), 16 de junio de 1814, p. 2; Western Citizen (Paris, KY), 2 de julio de 1814, pp. 1-2; Union (Washington, KY), 2 de julio de 1814, p. 1; Federal Republican (Georgetown, DC), 10 de junio de 1814, p. 3; Connecticut Journal (New Haven, CT), 13 de junio de 1814, p. 2; Connecticut Courant (Hartford, CT), 7 de junio de 1814, p. 3 y Connecticut Mirror (Hartford, CT), 13 de junio de 1814, p. 2 o Connecticut Gazette (New London, CT), 15 de junio de 1814, p. 2; Republican Star (Easton, MD), 14 de junio de 1814, p. 2; Baltimore Patriot (Baltimore, MD), 28 de abril y 10 de junio de 1814, pp. 3 y 2, y Gazette (Portland, ME), 6 de junio de 1814, p. 3.

30 Se transmitía que desde España «They speak with great indignation of this intrigue of Bonaparte», en New Hampshire Sentinel (Keene, NH), 2 de abril de 1814, p. 2. Ibid. en Sun (Dover, NH), 2 de abril de 1814, pp. 1-2; American and Commercial Daily Advertiser (Baltimore, MD), 31 de marzo de 1814, p. 2; Constitutionalist (Exeter, NH), 29 de marzo de 1814 p. 3; Concord Gazette (Concord, NH), 5 de abril de 1814 , p. 2; New Hampshire Sentinel (Keene, NH), 2 de abril de 1814, p. 2; Darmouth Gazette (Hanover, NH), 6 de abril de 1814, p. 2; o bajo titulares como Bonaparte's Intrigues with Spain, en Farmer's Cabinet (Amherst, NH), 28 de marzo y 11 de abril de 1814, pp. 2 y 2, y Weekly Advertiser (Kennebunk, ME), 2 de abril de 1814, p. 1.

31 Por ejemplo en Connecticut Gazette (New London, CT), 13 de abril de 1814, p. 1, Engine of Liberty and Uniontown Advertiser (Uniontown, MD), 7 de abril de 1814, p.2 o American and Commercial Daily Advertiser (Baltimore, MD), 30 de marzo de 1814, p. 3; Daily National Intelligencer (Washington, DC), 30 de marzo y 14 de abril de 1814, pp. 3 y 3 o Federal Republican for the Country (Georgetown, DC), 26 de abril de 1814, p. 4; Norwich Courier (Norwich, CT), 30 de marzo de 1814, p. 3 o Connecticut Mirror (Hartford, CT), 4 de abril de 1814, pp. 1-2. 
the cost of so much blood and treasure» ${ }^{32}$. Crítica que se une con la «imbecilidad» manifiesta de Fernando VII y los Borbones españoles a ojos de la opinión pública norteamericana y de la que se dará cuenta en el epígrafe siguiente.

Las noticias continúan ofreciéndose a partir de entonces en orden cronológico de sucesión. El 22 de marzo el rey entró en Valencia con el apoyo general del pueblo español, y para principios de mayo se multiplicaron las columnas al otro lado del Atlántico sobre la vuelta del monarca ${ }^{33}$. Se mostraba en todas ellas el entusiasmo del pueblo español tras conocer la noticia. Aunque, sin duda, lo que más espacio ocupó fue la promulgación del Decreto emitido por Fernando VII en el mes de mayo por el que declaraba nulas las competencias de las Cortes, y derogaba la Constitución gaditana ${ }^{34}$. En algún caso se expuso el texto completo, aunque la mayor parte de las veces apareció, de manera idéntica en todos los estados, un resumen traducido del mismo. La introducción de estas referencias señalaba el origen de las mismas en «the private armed Schooner Surprise, lately arrived at Newport, obtained at fen a file of Corunna papers, to May 21». Y se decía de esos «papeles» que «contain a long decree of Ferdinand $7^{\text {th }}$, issued at Valencia, the $4^{\text {th }}$ of May, 1814, declaring the Cortes of Spain and the Constitution formed under it, at and end». Especificando igualmente que el contenido reproducido en todos ellos «is a summary translation of this Important Document» ${ }^{35}$. Este resumen contenía alusiones históricas al inicio de la ocupación francesa y a la organización socio-política de España en ausencia del monarca ${ }^{36}$. Podrían destacarse estos fragmentos a modo de ejemplo para comprobar su contenido:

The Decree commences with a history of the proceedings of the French Government during the time they occupied Spain. -The king says, that on the $5^{\text {th }}$ May, 1808, he issued a Decree calling the Cortes, which continued their sittings until January, 1810, when the first Council of the Regency was called, which exercised their powers to September, 1810, when the General and Extraordinary Cortes was formed, and was installed in the Isle of Leon; and that at their first sitting the members of the Cortes, took an oath to preserve to him all his sovereignty. -The King then proceeds to give a history of the manner in which the Cortes were chosen, and says, that the Clergy and Nobles were

32 American and Commercial Daily Advertiser (Baltimore, MD), 31 de marzo de 1814, p. 2.

33 Una referencia en Constitutionalist (Exeter, NH), 3 de mayo de 1814, p. 3, entre otras muchas

34 Titular en Weekly Visiter (Kennebunk, ME), 30 de julio de 1814, p. 1, bajo el titular «Foreign Articles. From Spain. Newport, July 16. King Ferdinand refuses the New Constituion and dismisses the present Cortes».

35 Ejemplo de esa traducción son los fragmentos aparecidos en Palladium of Liberty (Morristown, NJ), 28 de julio de 1814, pp. 2-3; New Hampshire Patriot (Concord, NH), 26 de abril de 1814, p. 3; Sun (Dover, NH), 23 de julio de 1814, pp. 2-3, bajo titular «Rhode Island. Newport, July 16. From Spain-Late \& Interesting»; Darmouth Gazette (Hanover, NH), 27 de julio de 1814, pp. 2-3; Portsmouth Oracle (Portsmouth, NH), 23 de julio de 1814, p. 2; o Georgia Argus (Milledgeville, GA), 10 de agosto de 1814, p. 2; Western Monitor (Lexington, KY), 13 de agosto de 1814, p. 2 (donde se reproduce el decreto literal); Western Citizen (Paris, KY), 6 de agosto de 1814, p. 3; en Federal Republican (Georgetown, DC), 26 de julio de 1814, p. 3 y Federal Republican for the Country (Georgetown, DC), 29 de julio de 1814 p. 4 o Daily National Intelligencer (Washington, DC), 27 de julio de 1814, p. 3. Ibid. en Connecticut Journal, 25 de julio de 1814, p. 3 o American and Commercial Daily Advertiser (Baltimore, MD), 23 de julio de 1814, p. 2; Baltimore Patriot (Baltimore, MD), 22 de julio de 1814, p. 2; American Advocate (Hallowell, ME), 23 de julio de 1814, p. 3; Berkshire Reporter (Pittsfield, MA), 11 de agosto de 1814, p. 1; o New Jersey Journal (Trenton, NJ), 2 de agosto de 1814, p. 2.

36 American Advocate (Hallowell, ME), 23 de julio de 1814, p. 3, entre otras citas posibles. 
not called, although the Central Junta ordered it done: -That the first act of the Cortes was, to depose his sovereignty, attributing it to the nation, that they might appropriate it to themselves; and that this attempt against the prerogatives of the throne, was the basis of all their future acts. That almost all the forms of the ancient constitution of the monarchy were innovated upon, and that the Cortes copied the revolutionary and democratic principles of the French constitution of 17191; - that to prepare the minds of the people to receive these innovations, through the public prints, they abused the liberty of the press to make the Royal power odious, giving to the rights of Majesty the name of Despotism -making synonymous those of king and Despot, and calling Kings, Tyrants. -That they persecuted all those who opposed contradicted them, and in all things imitated a democrat - the King then proceeds:

'I declare that it is my Royal will and pleasure, not only not to swear to, nor accede to said Constitution, nor to any Decree of the General and Extraordinary, and of the Ordinary Cortes, now actually in session, who would deprive me of the rights and prerogatives of my sovereignty, established y the Constitution and the laws, under which the nation has long lived: but do declare that Constitution, and all such Decrees, null and void, and that they are from this time repealed, and without any obligation upon my people and subjects of whatever class or condition to comply with or regard them... ${ }^{37}$.

Tanto si se trataba de una reproducción íntegra del decreto como de un resumen, en todos los casos se anunciaba el reinado libre e independiente de Fernando VII, «as his ancestors had done ${ }^{38}$. Y a partir de entonces, coincidiendo en el tiempo en la retransmisión de la noticia, se aludía a la llegada del monarca y su reconocimiento como tal en una recepción aceptada por la mayoría, ya que muy pocos se opusieron en esos primeros días del mes de mayo al regreso de Fernando VII ${ }^{39}$. Sentimiento diferente

37 Tomada de Republican Farmer (Bridgeport, CT), 20 de julio de 1814, 2. Ibid. en Republican (Ohio), 15 de abril de 1814, p. 1 y American Whatchman (Wilmington, DE), 30 de julio de 1814, p. 2; Palladium of Liberty (Morristown, NJ), 28 de julio de 1814, 2; Carlisle Gazette (Carlisle, PA), 29 de julio de 1814, p. 2; Alexandria Gazette (Alexandria, VA), 30 de julio de 1814, p. 2; New-York Gazette (New Cork, NY), 21 de julio de 1814, p. 3, Long-Island Star (Brooklyn, NY), 27 de julio de 1814, p. 2, National Advocate (New York, NY), 22 de julio de 1814, p. 2, Geneva Gazette (Geneva, NY), 3 de agosto de 1814, p. 2 y New York Herald (New York, NY), 23 de julio de 1814, p. 1.

38 Literal en Palladium of Liberty (Morristown, NJ), 4 de agosto de 1814, p. 2 [«Spanish Affairs», Savannah, 9 de Julio] o Delaware Gazette and State Journal (Wilmington, DE), 1 de agosto de 1814, p. 3. Una introducción similar a la expuesta, pero con alguna variación en sus expresiones se reprodujo también en Gleaner (Wilkes-barre, PA), 5 de agosto de 1814, p. 1 y versa: «Immediately after the arrival of Ferdinand $7^{\text {th }}$ of Spain, at Valencia, he issued a Proclamation, dated May 4, 1814, declaring the dissolution of the General Cortes of Spain, and that the excellent Constitution which had been formed and ratified by the Spanish People, was at an end! This Proclamation, or Decree, is very elaborate. It gives a historical narrative of events, since the French occupied Spain, and of the organization of the Spanish political bodies, which took place in consequence. It states, that when the General and Extraordinary Cortes were held near Cadiz, the members swore «to preserve to him (Ferdinand $7^{\text {th }}$ ) all his sovereignty». He then proceeds to denounce all the proceedings of the Cortes - He says their first act was to depose him and assume, in the name of the nation, the Sovereignty; - that all the forms of the ancient constitution had been innovated upon by them; that the democratic principles of the French Constitution of 1791 had been copied into a Spanish one: - that the liberty of the press had been abused to make Royalty odious: and that all those who opposed this democratic order of things, had been persecuted: - He then proceeds: «I declare...». [Recibido por los editores del Newport Mercury en un documento español (Corunna) sobre los papeles del 21 de mayo].

39 Varios de los decretos mencionados aparecieron juntos, por ejemplo en Delaware Gazette and State Journal (Wilmington, DE), 8 de agosto de 1814, p. 2. 
sería el que comenzó a gestarse unos meses después de su llegada, cuando empezó a ponerse de manifiesto el fracaso de sus decisiones y la ineficiencia de su política.

Cabe señalar que cuando lo que se recibía en los puertos estadounidenses eran papeles, prensa o cartas directamente generadas por las autoridades españolas autorizadas se detectaba un tono claramente benévolo que mostraba cómo:

The King proclamation was read at the Puerta del Sol amidst the loudest acclamations. The people broke into the Sala de las Cortes, and without injuring the building erased the word Constitution, and removed the statue of Liberty. That figure was of wood and painted like bronze, an emblem, say the enemies of the Liberals, of the work they had erected -bold and impudent in appearance, really worthless in its material. It was conveyed by the mob to the Plaza Mayor beheaded and burnt. Rejoicings, acclamations, triumphal arches, and illuminations followed. The king was drawn into the streets by the people and the members of the Cortes, were carried to prison without the slightest appearance of concern or alarm in the metropolis. On the $6^{\text {th }}$ of May, before the revolution at Madrid, the people of Seville had risen against the Constitution, and proclaimed Ferdinand hereditary Sovereign of Spain and the Indies ${ }^{40}$.

Palabras como joy, congratulation o enthusiasm se repetían para explicar que «the feeling manifested by the inhabitants of the capital upon this occasion, affords a most satisfactory testimony of their unshaken loyalty attachment to their legitimate Sovereign ${ }^{41}$. Un sentimiento que se representó en repetidas ocasiones en expresiones de fidelidad y lealtad al rey que exhortaban al pueblo «True Sons of your Country! The moment has finally arrived of congratulating you upon the information that our beloved Monarch breathes, free from the heavy chains, that have oppressed him. If you lost him surrounded by enemies, you will recover him triumphant and full of glory» ${ }^{42}$. Un rey de quien se reconocía «the purity of his heart, and the rectitude of his intentions» ${ }^{43}$.

De la mano de esta versión de los acontecimientos en este lado del Atlántico, cuando se trató en Estados Unidos de reproducir noticias procedentes de las páginas de otros periódicos europeos o fruto de la redacción propia de los editores americanos, generalmente la visión que se ofreció fue más crítica y, sobre todo, menos uniforme y positiva. Se encuentran entonces manifestaciones y testimonios que aluden al grado de descontento de muchos españoles por el viraje de los sucesos políticos. Un males-

40 Star (Raleigh, NC), 14 de agosto de 1814, p. 130. Similar en New Jersey Journal (Elizabethtown, NJ), 19 de abril de 1814, p. 1; Federal Republican (Georgetown, DC), 15 de agosto de 1814, p. 2; New Bedford Mercury (New Bedford, MA), 12 de agosto de 1814, p. 2; New England Palladium (Boston, MA), 5 de agosto de 1814, p. 2; Boston Daily Advertiser (Boston, MA), 6 de agosto de 1814, p. 2; Star (Raleigh, NC), 19 de agosto de 1814, p. 130; Gleaner (Wilkes-barre, PA), 26 de agosto de 1814, p. 3; Poulson's American Daily Advertiser (Philadelphia, PA), 9 de agosto de 1814, p. 2 o Washington Reporter (Washington, PA), 12 de septiembre de 1814 , p. 1 .

41 Constitutionalist (Exeter, NH), 14 de junio de 1814, p. 4. También Daily National Intelligencer (Washington, DC), 13 de junio de 1814, p. 2; Federal Republican (Georgetown, DC), 10 de junio de 1814, p. 3; Gazette (Portland, ME), 6 de junio de 1814; o Hallowell Gazette (Hallowell, ME), 8 de junio de 1814, p. 3.

42 Sun (Dover, NH), 9 de abril de 1814, pp. 1-2; Western Citizen (Paris, KY), 30 de abril de 1814, p. 1; Connecticut Herald (New Haven, CT), 12 de abril de 1814, p. 2; American and Commercial Daily Advertiser (Baltimore, MD), 11 de abril de 1814, p. 2; Hallowell Gazette (Hallowell, ME), 13 de abril de 1814, p. 2.

43 Palabras de Delaware Gazette and State Journal (Wilmington, DE), 1 de agosto de 1814, p. 1. 
tar entre el pueblo español fruto de las tensiones que «still subsist between Ferdinand VII and the Cortes of Spain». Y que llevaban a pensar que «it would be a lamentable thing if a civil war should break out in that country $\rangle^{44}$. Lejos de la imagen oficial de orden y equilibrio que pretendía transmitirse, según estas otras fuentes la situación real era de inestabilidad y confusión ${ }^{45}$. Y era precisamente esta perspectiva la que explicaría iniciativas como la Espoz y Mina, vistas como «an opposition to Ferdinand, with twelve thousand men»; a las que se creía se unirían «the old adherents of the Cortes system of administration $\rangle^{46}$.

A pesar de que en un cómputo total predominan los artículos que transmitieron las «noticias oficiales», lo cierto es que los estadounidenses no debieron tener una imagen desdibujada de la realidad española, ya que estas últimas alusiones a los aspectos menos «amables» del retorno del absolutismo estuvieron igualmente muy presentes. Así, desde Londres llegaron la mayor parte de referencias a la persecución generalizada que el monarca estaba llevando a cabo contra liberales y afrancesados desde su regreso al trono. Durante el mes de septiembre se reiteraron las noticias procedentes de Gran Bretaña que hacían hincapié en esa idea ${ }^{47}$, y que mostraban cómo «the situation of Spain was deplorable indeed; a complete and thorough revolution had been effected, without any public commotion, and Ferdinand is now firmly seated on the throne, and is more despotic than ever Bonaparte was. He has abolished the Constitution and all the good and beneficial laws established by the Cortes» ${ }^{48}$.

Pero no sólo del resto de Europa llegaron informaciones en esos términos. Algunas confidencias procedentes de Cádiz, bastión liberal en el primer periodo constitucional de España, reclamaban el posicionamiento de una gran parte de la población en contra de la restauración de Fernando VII, bajo el grito No Bonapartes - No Bourbons ${ }^{49}$. En esta misma línea, que además justifica la crítica internacional hacia el monarca, alguno de los individuos que llevaron consigo a Estados Unidos cartas y experiencias personales presentaron ese panorama de lucha y disconformidad, muy diferente a la unanimidad positiva y «feliz» de la versión oficial del nuevo gobierno:

European Accounts. A letter from a gentleman who left Cadiz the 28th July, states that the situation of the country was found to be truly deplorable; that a through revolutions had been effected and Ferdinand finally seated on the throne, with more despotic power than Bonaparte ever assumed; that he had entirely abolished the constitution, with all the beneficial laws established by the Cortes; imprisoned great numbers of

44 Farmer's Cabinet (Amherst, NH), 1 de agosto de 1814, p. 1. Cit. también en Federal Republican (Georgetown, DC), 25 de julio de 1814, p. 3 o Middlessex Gazette (Middletown, CT), 28 de julio en 1814, p. 2. Similar en Baltimore Patriot (Baltimore, MD), 23 de julio de 1814, p. 2; American Advocate (Hallowell, ME), 30 de julio de 1814, p. 2.

45 Detalle en American and Commercial Daily Advertiser (Baltimore, MD), 25 de julio de 1814, p. 3.

46 Sun (Dover, NH), 12 de noviembre de 1814, p. 3. También en Western Citizen (Paris, KY), 29 de octubre de 1814 , p. 3 .

47 En la sección de Foreign News de numerosos periódicos. Entre otros en Star (Raleigh, NC), 16 de septiembre de 1814, p. 146 y American Watchman (Wilmington, DE), 10 de septiembre de 1814, p. 2.

48 Así expresado en Federal Republican for the Country (Georgetown, DC), 7 de octubre de 1814, p. 1 o Boston Daily Advertiser (Boston, MA), 29 de septiembre de 1814, p. 3, por citar únicamente dos ejemplos.

49 Hallowell Gazette (Hallowell, ME), 6 de julio de 1814, p. 3. 
its members, as well as many public officers and citizens; the worst of all, had reestablished the Inquisition ${ }^{50}$.

Aunque es necesario hacer notar que son noticias reproducidas en el mes de octubre de 1814, momento en que se han empezado a notar los efectos del restablecimiento del poder absoluto del rey y esa dura represión contra los elementos liberales del país, contra aquéllos que habían ofrecido su apoyo a José I Bonaparte, y contra los militares y sus familias que habían servido a aquél en su ausencia ${ }^{51}$.

De cualquier modo, lo cierto es que derivada de ambos posicionamientos sociales e ideológicos, se proyecta en los periódicos estadounidenses de 1814 la realidad de una España en la que convivían el clima general de aceptación al regreso del monarca y una clara oposición de los sectores más avanzados y fieles al liberalismo que pretendían seguir luchando para lograr su triunfo definitivo ${ }^{52}$.

\subsection{El contenido de opinión de sus artículos}

Junto con esa traducción, literal o libre, más o menos objetiva, de las noticias que llegaban desde Europa, se pudo observar la opinión crítica que editores y articulistas transmitieron en sus columnas; lo cual permite a su vez tener una imagen más completa de la idea de España que en 1814 pudieron forjarse los estadounidenses a través de sus periódicos. Así como las transcripciones emitían los comunicados públicos llegados desde la Península, estas otras eran realmente «in which newspapers most directly pronounce their views $\rangle^{53}$.

El menor número de esas referencias críticas en sus páginas se explica por la propia naturaleza de la prensa a principios del siglo XIX, y por el hecho de que:

These political journals, circulating among a small and very political-minded class, would even under favorable conditions have been slow to range outside the political

50 En Farmer's Cabinet (Amherst, NH), 3 de octubre de 1814, p. 3. En Norwich Courier (Norwich, CT), 5 de octubre de 1814, p. 2 ese fragmento se complementa con la idea «He had also levied a contribution of 150 million dollars on his subjects, which, from the impoverished state of the country, could not be collected, but by the most arbitrary and cruel exactions». En esos mismos términos se expresa el artículo «Important from lake Champlain», en American and Commercial Daily Advertiser (Baltimore, MD), 4 de octubre de 1814, p. 2 o «European Accounts» en Hallowell Gazette (Hallowell, ME), 5 de octubre de 1814, p. 3 y Northern Post (Salem, NY), 27 de octubre de 1814, p. 3.

51 Esta idea aparece por ejemplo en Norwich Courier (Norwich, CT), 17 de agosto y 21 de septiembre de 1814 p. 2; y Connecticut Gazette (New London, CT), 21 de septiembre de 1814, p. 3; Connecticut Courier (Bridgeport, CT), 3 de agosto de 1814, p. 2; o American Advocate (Hallowell, ME), 17 de septiembre de 1814, p. 3.

52 Un ejemplo que combina ambos aspectos es la reflexión siguiente: «el trono es en España la base de todo gobierno: las instituciones liberales no pueden conservarse sino apoyadas en él, unámonos con él estrechamente para que la libertad crezca a su sombra». Palabras de D. Ramón Alesón Alonso de Tejada (1781-1846) en el Archivo Histórico Provincial de La Rioja, Fondo P-A, caja 032/18, proclamas dirigidas a los electores de la provincia de Logroño. Cit. en Rebeca Viguera Ruiz, «El moderantismo en la etapa postrevolucionaria. Un ejemplo en clave local a comienzos del siglo», Historia Constitucional, $\mathrm{n}^{\circ}$ 14, 2013, 173198.

53 KAPLAN, Richard L.: Politics and the American Press. The Rise of Objectivity, 1865-1920. CambridgeUnited Kingdom: Cambridge University Press, 2002, p. 27. 
field. But conditions were anything but favorable. It was impossible to present a varied editorial fare in an era which lacked varied social and intellectual interests. A raw young nation which had no independent literature, no drama, no art, no music, intent upon conquering a living from the wild forest and prairie. [...] Indeed, one function of the editorial columns until the thirties was to furnish news regarding even events of the greatest importance ${ }^{54}$.

Era una prensa joven, en auge, con un carácter fuertemente político, centrada en los debates internos de sus instituciones y poderes públicos, y más preocupada por generar una opinión pública nacional que por cuestiones internacionales que no estuvieran directamente relacionadas con sus intereses. En aquellas escasas columnas donde se pueden apreciar matices más concretos sobre la figura de Fernando VII predomina su presentación como un tirano que había terminado por arruinar la causa política liberal, peninsular e hispanoamericana, «by disavowing the Constitution drawn up by the Junta» ${ }^{55}$. Una actitud inesperada que suscitaba el desencanto y la sorpresa de muchos y que, según los redactores estadounidenses, se había generado a causa de un monarca «very ill advised since his return» ${ }^{56}$, que había llevado «the excellent constitution which had been formed and ratified by the Spanish people, at an end!» ${ }^{57}$.

En realidad coincidían en afirmar que los sucesos desarrollados tras el regreso de Fernando VII no hacían sino restablecer un sistema de gobierno anticuado, que se había forjado en tiempos de los Reyes Católicos en el siglo XV y que ya no se correspondía ni con las necesidades del pueblo español ni con la lucha liberal que se estaba produciendo a nivel internacional. Lo cual «represents the people to be wholly insensible to the internal despotism establishing others give the most deplorable

54 NEVINS, Allan: American Press opinion..., op. cit., p. 6. Complemento de esta obra, entre otras, es la de REEVES, Richard: What the People know. Freedom and the Press. Cambridge: Massachusetts, Harvard University Press, 1998.

55 New Jersey Journal (Elizabethtown, NJ), 1 de noviembre de 1814, p. 3 o similar en Ibid. 18 de octubre de 1814 , p. 4.

56 Esas ideas se ponen de manifiesto tras la recepción de noticias que situaban a Fernando VII en Valencia, antes de su llegada a Madrid, y tras conocer el contenido del decreto de derogación de la Constitución. Ejemplos en Western Monitor (Lexington, KY), 23 de septiembre de 1814, p. 1; Baltimore Patriot (Baltimore, MD), 7 de septiembre de 1814, p. 2; Union (Washington, KY), 6 de agosto de 1814, p. 3 y Daily National Intelligencer (Washington, DC), 28 de julio de 1814, p. 3; Norwich Courier (Norwich, CT), 10 de agosto de 1814, p. 2; American and Commercial Daily Advertiser (Baltimore, MD), 20 de julio de 1814, p. 3; Palladium of Liberty (Morristown, NJ), 4 de agosto de 1814, p. 2; New-Hampshire Gazette (Portsmouth, NH), 9 de agosto de 1814, p. 2; New-Hampshire Patriot (New Hampshire, NH), 9 de agosto de 1814, p. 2, Boston Gazette (Boston, MA), 4 de agosto de 1814, p. 2 y Poulson's American Daily Advertiser (Philadelphia, PA), 9 de julio de 1814, p. 3; Enquirer (Richmond, VA), 30 de julio de 1814, p. 3; New-York Herald (New York, NY), 3 de agosto de 1814, p. 2; Columbian (New York, NY), 29 de julio de 1814, p. 3; Long-Island Star (Brooklyn, NY), 3 de agosto de 1814, p. 3; Evening Post (New York, NY), 30 de julio de 1814, p. 3, New-York Gazette (New York, NY), 30 de julio y 1 de agosto de 1814, pp. 3 y 3; New Jersey Journal (Elizabethtown, NJ), 6 de septiembre de 1814, p. 3; New Hampshire Patriot (Concord, NH), 9 de agosto de 1814, p. 2 o Portsmouth Oraclek (Portsmouth, $\mathrm{NH}), 10$ de septiembre de 1814 , p. 1

57 Manteniendo la cursiva del original, en New Jersey Journal (Elizabethtown, NJ), 2 de agosto de 1814, pp. 2-3 y Norwich Courier (Norwich, CT), 27 de julio de 1814, p. 2. 
accounts of the state of affairs, and represent the unhappy country to be on the eve of great commotions $\rangle^{58}$.

En medio de esa representación, «mad tyrant» ${ }^{59} \mathrm{y}$ «miserable fanatic» son dos de los adjetivos que más acompañaron en este momento al nombre del monarca español. Un fanático absolutista que no dudó en anular «the constitution which had been framed to restore the rights of the people», que «usurped upon their rights and gradually contrived to annihilate them ${ }^{60}$. Una visión más claramente negativa de su figura y de las repercusiones que su restauración monárquica conllevaba para el país. Con ella desaparecían todas las esperanzas del pueblo de recuperar sus derechos y libertades y de disfrutar de ellos. Pero a su vez suponía un claro y duro golpe para la realidad política Europea y sus expectativas liberales. En esa misma referencia el Enquirer se lamentaba: «Deluded Europe! When will you discover that one man is born equal to another; and that government were instituted for the good of your people, and not for the good of your Kings? $\rangle^{61}$. Al mismo tiempo que Europa y España retrocedían hacia postulados políticos propios de un Antiguo Régimen todavía en parte latente en los tronos absolutistas, los Estados Unidos de América luchaban en defensa de su libertad, por constituirse como nación y por hacer prevalecer una democracia que había iniciado sus primeros pasos tras la independencia ${ }^{62}$.

De ahí que esa tiranía de Fernando VII le hiciese aparecer, en los periódicos norteamericanos, como un «bigotted, tyrannical prince», de manifiesta imbecilidad ${ }^{63}$, $\mathrm{y}$ «unworthy the affection of a people who have made such sacrifices» ${ }^{64}$. Aquel que como un déspota sin escrúpulos, «for he is not deserving no other appellation», decidió ir en contra de todos aquellos que «openly thed their blood for him and his family» ${ }^{65}$.

Él «had declared the New Spanish Constitution to be null and void», y además había también «arrested and banished many of the Functionaries and had appointed, and reinstated most of the Ancient Nobles to their offices [...] with al the rights, ho-

58 New Hampshire Sentinel (Keene, NH), 8 de octubre de 1814, p. 3; New Hampshire Gazette (Portsmouth, NH), 11 de octubre de 1814, p. 1; American and Commercial Daily Advertiser (Baltimore, MD), 10 de octubre de 1814 , p. 2.

59 Así descrito en el artículo que presenta la incoherencia del restablecimiento de la Inquisición y del absolutismo que podía tener su origen con los Reyes Católicos, bajo el titular «European News», en New Hampshire Sentinel (Keene, NH), 8 de octubre de 1814, p. 3. Similar noticia en Sun (Dover, NH), 8 de octubre de 1814, p. 2; Gazette (Pórtland, ME), 10 de octubre de 1814, p. 2.

60 Missouri Gazette and Public Advertiser (St. Louis, MO), 10 de septiembre de 1814, p. 2.

61 Ibid. p. 3.

62 Todo ello en una situación histórica compleja de estructuración política y configuración intelectual e ideológica de la identidad norteamericana que no se trata aquí de manera exhaustiva por responder en sí misma a objeto de un estudio mucho más amplio en comparativa con la evolución socio-política coetánea en el marco del mundo occidental. Al respecto pueden consultarse como punto de partida los trabajos de Gordon S. Wood, The Radicalism of the American Revolution, New York, A.A. Knopf, 1992, y Empire of Liberty: A History of the Early Republic, 1789-1815, USA, Oxford University Press, 2009.

63 American and Commercial Daily Advertiser (Baltimore, MD), 31 de marzo de 1814, p. 2.

64 Entre otras citas, aparece referenciado en New Hampshire Sentinel (Keene, NH), 23 de julio de 1814, p. 3.

65 Por ejemplo en New Hampshire Sentinel (Keene, NH), 1 de octubre de 1814, p. 3. 
nours and prerogatives» ${ }^{66}$. Incluso «that poor, miserable, infatuated bigot, Ferdinand of Spain, has, since the re-establishment of the Inquisition, commenced a persecution against the Free Masons, many of whom have been arrested and delivered over to torture and death ${ }^{67}$. Persecución que, junto con la ejecutada contra los liberales, justificaba las críticas contra su actuación. Y no dejaba de sorprender el hecho de que todo ello se había producido «not only been done by the full consent, but by the absolute demand of the nation ${ }^{68}$. Lo cual, desde la perspectiva de la prensa que se está analizando, no evitaría que «a day of retribution will come when this monster, this prototype of Philip the $2^{\text {nd }}$, will meet the reward of his crimes» ${ }^{69}$.

Por lo tanto se perfiló a los ojos de la prensa internacional un personaje insolidario que no dudó en obviar los sacrificios que su pueblo había realizado en defensa de su trono $^{70}$. Un soberano que, «next to the Legitimates of Portugal, the Royal family of that country were the most notorious for their imbecility and total destitution of talent $\rangle^{71}$. Fueron palabras de un elevado contenido crítico y mordaz, muy directas e incisivas; reflejo de las conclusiones que se habían extraído tras el conocimiento de los sucesos acaecidos en España.

\section{El desencanto liberal de Estados Unidos ante la supresión de La Pepa}

Cuando a mediados de 1812 se conoció la noticia de la proclamación de la Constitución de Cádiz de 19 de marzo, la Pepa, y su apuesta por los derechos y libertades que se barajaban en el ámbito de las revoluciones liberales, España se convirtió en ejemplo de superación, patriotismo y lucha por la libertad ${ }^{72}$. Desde Estados Unidos

${ }^{66}$ American Watchman (Wilmington, DE), 30 de julio de 1814, p. 2 o Western Courier (Louisville, KY), 15 de agosto de 1814, p. 2.

67 Centinel of Freedom (Newark, NJ), 13 de diciembre de 1814, p. 3 y Connecticut Herald (New Haven, CT), 6 de diciembre de 1814, p. 3; o Delaware Gazette and State Journal (Wilmington, DE), 1 de diciembre de 1814, p. 1 [del Freeman's Journal]. Numerosas críticas se derivaron de ese restablecimiento del Tribunal de la Inquisición, que según palabras del monarca, se sentenció con el fin de asegurar y perpetuar el poder de la Iglesia y la fe católica entre los españoles. Última reflexión en Western Courier (Louisville, KY), 2 de noviembre de 1814, p. 2 y Connecticut Herald (New Haven, CT), 8 de octubre de 1814, p. 1.

68 Ibid nota 59.

69 Centinel of Freedom (Newark, NJ), 13 de diciembre de 1814, p. 3. Firmado por Phil F. Tour.

70 Connecticut Spectator (Middletown, CT), 28 de septiembre de 1814, p. 1. Un dato concreto sobre ello se observa en la orden de detener a los editores de El Redactor y El Conciso, "who with great energy maintained the cause of their country against its atrocious invaders, and without whose strenuous efforts the throne of the ungrateful Ferdinand might at this moment have been occupied by a stranger». Reproducido en American and Commercial Daily Advertiser (Baltimore, MD), 2 de agosto de 1814, p. 2; Boston Daily Advertiser (Boston, MA), 28 de julio de 1814, p. 2; Repertory (Boston, MA), 29 de julio de 1814, p. 1; Staunton Observer (Staunton, VA), 18 de agosto de 1814, p. 2; Virginia Patriot (Richmond, VA), 10 de agosto de 1814, p. 4; Columbian Gazette (Utica, NY), 9 de agosto de 1814, p. 2; Orange County Patriot or The Spirit of Seventy-six (Goshen, NY), 9 de agosto de 1814, p. 1 o Columbian (New York, NY), 30 de julio de 1814, p. 1 , entre otros.

71 Literal de American and Commercial Daily Advertiser (Baltimore, MD), 16 de julio de 1814, p. 3.

72 New-Hampshire Patriot (New Hampshire, NH), 1 de enero de 1812, p. 1; Federal Gazette (Baltimore, MD), 29 de diciembre de 1812, p. 2; Albany Gazette (Albano, NY), 21 de septiembre de 1812, p. 2. Referencias en VIGUERA RUIZ, Rebeca: «El triunfo constitucional español de 1812 en las páginas de la prensa norteamericana». PILAR. Prensa, Impresos, Lectura en el Área Románica, 2013, pp. 37-48. 
se destacó el coraje español como inspiración y modelo a seguir en la defensa de los derechos de la humanidad. Debía imitarse y admirarse el espíritu nacional y patriótico de un pueblo determinado a ser libre que no permitiría de nuevo ser esclavizado ${ }^{73}$. Y en medio de ese panorama, se resaltaba que «the new Constitution by which the condition of the People of Spain is greatly ameliorated, the Inquisition annihilated, and the powers of the Nobles and Clergy limited, has been every where received with gratitude and ratified with festive acclamations» ${ }^{74}$.

La restitución del absolutismo en 1814 suponía no sólo la eliminación o derogación de la constitución, sino también de los derechos y libertades vinculados a ella. Significaba pasar por alto y menospreciar todos los esfuerzos y sacrificios del pueblo español desde 1808. Y, ante todo, suponía una amenaza para las aspiraciones liberales del panorama internacional a principios del siglo XIX.

Desde la perspectiva norteamericana la decepción ante el giro de los acontecimientos era manifiesta, como se ha podido comprobar en las descripciones que concedieron al propio Fernando VII. Después de que el mundo hubiera sido testigo del coraje y éxito de los españoles, no se podía creer que «the reign of despotism, bigotry and priest craft can never be restored in Spain $\rangle^{75}$. Parecía imposible que tras su regreso todas sus expectativas quedaran truncadas.

\subsection{Decepción y rechazo ante el regreso del absolutismo al panorama político español}

Ante la proliferación de noticias sobre la decisión de Fernando VII de no ratificar el texto constitucional, de encarcelar a más de treinta individuos adeptos al régimen liberal del gobierno provisional, y de manifestar su deseo de reinar libremente de acuerdo a los viejos principios monárquicos, la incredulidad ante la posibilidad de que el absolutismo pudiera volver a tener cabida en la política y la sociedad de España se incrementaba tras las recepción de noticias que aseguraban que «the people in general (some provinces excepted) are in favour of the constitution, and have sufficient proof of the good effects already experienced from it, to support it. [...] I am of opinion, most decidedly, that the liberty of the press has already opened the eyes of the people sufficiently to prevent them giving up their rights without at least making a struggle for them $\rangle^{76}$.

La realidad que se vivió en el país durante el sexenio absolutista posterior, demostraría lo inexacto de esas esperanzas previas. Más bien al contrario:

The restored tyrant of Spain is taking every measure to render his people the most ignorant, that they may be the most confirmed and degraded of slaves. After prostra-

73 Expresiones extraídas de American and Commercial Daily Advertiser (Baltimore, MD), 29 de enero de 1812, p. 2.

74 Ejemplos en la Boston Gazette, 28-IX-1812, p. 2 y Weekly Messenger, 2-X-1812, p. 4, Spooners Vermont Journal, 5-X-1812, p. 3, o la Concord Gazette, 6-X-1812, p. 1.

75 American Watchman (Wilmington, DE), 30 de julio de 1814, p. 2.

76 «Extract of a letter from Cadiz, 8th february», en Federal Republican (Georgetown, DC), 3 de mayo de 1814, p. 2 y Poulson's American Daily Advertiser (Philadelphia, PA), 29 de abril de 1814, p. 3. 
ting the liberty of the Spanish press, he has prohibited the circulation (or perusal) of foreign papers in his kingdom. Thus is Spain fast emerging to the darkness of barbarian ages. The only rays of intellectual light which se is to enjoy, are the destroying flames of that 'hell upon the bosom of the earth', the Inquisition. Modern times do not furnish a parallel to the despotism of Ferdinand, and we question whether the enlightened state of the world will suffer it long to exist. We hope for the honor of mankind, and the happiness of the Spanish nation, that the reign of the tyrant may be short ${ }^{77}$.

Esta misma idea se repite en más de una ocasión con diferentes expresiones, pero siempre haciendo hincapié en las injusticias sociales ocasionadas por el restablecimiento de la Inquisición, la abolición de las Cortes y la Constitución, y la prohibición de todo tipo de información pública que pudiera transmitirse en el país de manera libre y sin previa censura. El Palladium of Liberty consideraba que debía existir en España en ese momento «a general dissatisfaction» que conllevaría la espera, por el honor del mundo civilizado y la libertad de la humanidad, «that the existent of such a tyranny is to be suffered but for a short period $\rangle^{78}$. O incluso un individuo norteamericano, que había vivido en España durante los últimos tres años, aseguraba que desde la perspectiva del momento el nuevo sistema de gobierno establecido por Fernando VII no podía tener una duración larga en el tiempo. Aquél «will either be assassinated or compelled to abdicate his throne; his conduct has been so unexpected that the people are panic struck, and want a bead to lead them on to a maintenance of the principles of the New Constitution ${ }^{79}$. Como se mencionaba unas líneas más arriba, la evolución posterior de la historia española mostraría una realidad muy diferente.

De cualquier modo, para un país pionero en la búsqueda de derechos y libertades individuales para sus ciudadanos e instituciones, la anulación de las Cortes y organismos políticos de corte liberal, así como toda la obra constitucional iniciada en 1812 y sus principios liberales, supuso un desencanto notable. En el plazo de pocas semanas se acabó con el significado democrático y liberal de aquéllas a causa de su parecido a los ideales que guiaron la Revolución Francesa. Ideales que harían posible una nueva realidad política y social, admirada por los Estados Unidos ${ }^{80}$, pero que a ojos de Fer-

77 Cursiva del original en New Jersey Journal (Elizabethtown, NJ), 18 de octubre de 1814, p. 4.

78 Palladium of Liberty (Morristown, NJ), 10 de noviembre de 1814, p. 3.

79 «Extract of a letter, dated Fernandina (Amelia Island), Sept. 8», reproducido en American Watchman (Wilmington, DE), 1 de octubre de 1814, p. 2; Federal Republican for the Country (Georgetown, DC), 23 de septiembre de 1814, p. 3; bajo titular «From the South» en Republican Farmer (Bridgeport, CT), 5 de octubre de 1814, p. 4, y tras el título «Foreign Intelligence» en Norwich Courier (Norwich, CT), 5 de octubre de 1814, p. 2. Esa misma noticia también en Connecticut Herald (New Haven, CT), 4 de octubre de 1814, p. 2; Columbian Register (New Haven, CT), 4 de octubre de 1814, p. 3; Engine of Liberty and Uniontown Advertiser (Uniontown, MD), 29 de septiembre de 1814, p. 3, Daily and Commercial Daily Advertiser (Baltimore, MD), 24 de septiembre de 1814, p. 2; Boston Patriot (Boston, MA), 1 de octubre de 1814, p. 2; New-England Palladium (Boston, MA), 30 de septiembre de 1814, p. 2; Providence Gazette (Providence, RI), 1 de octubre de 1814, p. 2, Weekly Aurora (Philadelphia, PA), 12 de abril de 1814, p. 409; Poulson's American Daily Advertiser (Philadelphia, PA), 26 de septiembre de 1814, p. 3 o Virginia Patriot (Richmond, VA), 1 de octubre de 1814, p. 1. En ese mismo sentido el Yankee predecía en agosto de 1814 que «that patriotism, and the spirit of liberty will prostrate FERDINAND as low as ever he was when a state prisoner in France», en Yankee (Boston, MA), 5 de agosto de 1814, p. 3.

80 En esos momentos «the Federal government merely provided the glue that kept the states from flying apart», buscando el modo de que la nueva nación fuera «a symbol of the broad consensus for democratic 
nando VII permitían prerrogativas socio-culturales y políticas excesivas que alejaban al gobierno y al pueblo del bien común ${ }^{81}$. Estas afirmaciones presentes en los decretos del monarca, dejaban atónitos a los espectadores internacionales:

These facts, we repeat, astonish us. We have not expected, that the Spanish People have been making efforts to beat down one tyrant merely to build up an other -even if legitimate. And we have had confident hopes that a well balanced government would have been the reward of Spanish valor, perseverance and loyalty. We have no, however, recently been without our fears. Ever since the treaty concluded between Ferdinand and the fallen Napoleon, was signed, that conduct of the former has worn a mask of mystery. [...] And while the Spaniards were daily expecting his entrance into Madrid, to take the oaths to support the Constitution, he fulminates his decree to abolish not only that Constitution, and the Cortes which free people had chosen; but assumes by himself the exercise of all the functions of ancient Monarchy! ${ }^{82}$.

De este modo los ifs and buts, los temores manifestados en la prensa norteamericana en el año $1812^{83}$, se habían confirmado mucho antes de lo esperado con la disolución del texto constitucional. Lo que anticipaba que «thus, at one blow, has the new constitution of Spain, been leveled in the dust -and we may expect, as soon as Louis the $18^{\text {th }}$ is firmly seated on his throne, that the new Constitution of France will share the same fate». Ante ese estado de cosas se lamentarían las páginas norteamericanas en torno al mes de julio de 1814 de cómo «such are the proceedings of the legitimate Sovereigns of Europe!» ${ }^{84}$. En el mes de agosto esas reflexiones se repetirían con líneas como las que siguen:

De ésta y otras citas similares se desprende la conclusión de que mientras casi todas las demás naciones de la faz de Europa, en un estado de regeneración suave y saludable, se preparaban para gozar de las bendiciones de un largo descanso bajo esas circunstancias..., España, envilecida, había dado su consentimiento para no volver a gozar de ello! Había invitado, abrazado, el antiguo despotismo de su monarca y sus sacerdotes ${ }^{85}$.

A pesar toda la sangre derramada en aquel país y los esfuerzos de su nueva clase política por instaurar nuevos principios y valores liberales ${ }^{86}$, la realidad era esa, una aceptación resignada de la restauración absolutista de su monarca legítimo. Había por ello un gran escepticismo general al otro lado del Atlántico sobre lo que estaba

principles». Una necesidad de consenso, libertad y lucha por sus principios políticos todavía presente en 1814. Reflexión en DICKERSON, Donna Lee: The course of Tolerance. Freedom of the Press in Nineteenth-century America. Westport, Connecticut: Greenwood Press, 1990, pp. XIII-XIV.

81 Ejemplo de esta expresión en New Hampshire Sentinel (Keene, NH), 23 de julio de 1814, p. 3.

82 En Western Courier (Louisville, KY), 15 de agosto de 1814, pp. 1-2; Norwich Courier (Norwich, CT), 27 de julio de 1814, p. 2; Middlessex Gazette (Middletown, CT), 28 de julio de 1814, pp. 2-3; Republican Farmer (Bridgeport, CT), 27 de julio de 1814, p. 2, o Providence Patriot (Providence, RI), 23 de julio de 1814 , p. 3.

83 Vide en VIGUERA RUIZ, Rebeca: «El triunfo constitucional español...», op. cit.

84 Todas expresiones tomadas de American Watchman (Wilmington, DE), 30 de julio de 1814, p. 2 y American and Commercial Daily Advertiser (Baltimore, MD), 23 de julio de 1814, p. 3

85 Trad. de American and Commercial Daily Advertiser (Baltimore, MD), 15 de agosto de 1814, p. 2.

86 Idea literal de Daily National Intelligencer (Washington, DC), 28 de julio de 1814, p. 2. 
sucediendo y, en definitiva, no se podía afirmar sino que «whether this change, in the Spanish government, will or will not be followed by civil commotion, a few months may decide» ${ }^{87}$.

\subsection{Repercusión en las colonias}

Una última reflexión en torno a estas cuestiones tiene que ver con el modo en que se transmitió desde Norteamérica la recepción del decreto de derogación de la Constitución de 1812 en las colonias españolas que más proximidad o relación tenían con los Estados Unidos, es decir, los territorios de Cuba, y aquellas zonas que permanecían bajo dominio español en el actual estado de Louisiana y la cuenca del Missisipi ${ }^{88}$.

En general las colonias se habían solidarizado con los peninsulares a partir de 1808 al tener noticia de la invasión francesa y el cautiverio de Fernando VII, constituyendo órganos de poder propio y reivindicando su igualdad de derechos. Al alborozo inicial que supuso aquella noticia siguió la configuración de grupos de tendencia liberal que, sin llegar a ser partidos políticos propiamente dichos, compartían objetivos comunes y un programa basado en la soberanía nacional, el estado de derecho, la igualdad jurídica y la representación popular ${ }^{89}$.

En ese contexto definido por la ocupación extranjera, la falta de un poder real en el trono y la ausencia de órganos de gobierno capacitados, la elaboración y proceso de la constitución gaditana influyó de manera directa en aquellos territorios. Tuvo entonces un especial impacto en el desarrollo histórico posterior de las colonias y la metrópoli el abandono de la organización estamental, la sustitución de la vieja monarquía absoluta y, por encima de todo ello, la existencia de una Constitución liberal por primera vez en los territorios de ultramar. Se sentaron las bases del constitucionalismo, la representación parlamentaria, la soberanía nacional y la división de poderes. Lo que supuso para la América hispana un valioso instrumento ideológico que permitió a la elite colonial reafirmar su poder social y político bajo un nuevo lenguaje democrático.

Por todo ello, la ratificación de los artículos de La Pepa supuso un motivo de alegría compartida. «The new constitution of Spain and her Indies arrived last month, and has been proclaimed with great ceremony and rejoicing» ${ }^{90}$. «By the new Constitution of Spain, the Spanish Colonies are placed upon the same footing, in relation to the mother country. They are to have their Legislative Assemblies, and other Executive Officers $\gg{ }^{91}$. Se entendió que las colonias gozarían de los mismos derechos que la metrópoli, de ahí la recepción de la noticia con «loud acclamation». Una satisfacción que se reflejó con «much music, much discharge of artillery, much splendour, pomp

87 American Watchman (Wilmington, DE), 6 de agosto de 1814, p. 3; o en Columbian Register (New Haven, CT), 2 de agosto de 1814, p. 3, y Massachusetts Spy (Worcester, MA), 20 de julio de 1814, p. 3.

88 Del resto de territorios coloniales no aparece alusión directa en los artículos estudiados.

89 Sobre estas idea más en BREÑA, Roberto: «El primer liberalismo español y la emancipación de América: tradición y reforma». Revista de Estudios Políticos (Nueva época), no 121, 2003, pp. $257-289$.

90 Extraído de una carta llegada de la Habana al estado District of Columbia el 16 de agosto de 1812. Apareció en Federal Republican (Georgetown, DC), 7 de septiembre de 1812, p. 3, y se reprodujo en Republican Star (Easton, MD), 8 de septiembre de 1812, p. 2, y New-England Palladium (Boston, MA), 11 de septiembre de 1812 , p. 2, entre otros.

91 New-England Palladium (Boston, MA), 20 de noviembre de 1812, p. 1. 
and ceremony» en los territorios coloniales en 1812. Cundió una "great illumination» entre los colonos «on account of the publication of the new Spanish constitution»" a la vista de las ventajas y derechos que con ella se adquirían.

Sin embargo, el tiempo demostraría que aquellos primeros planteamientos liberales carecían de un proyecto común para tratar la cuestión americana. Lafit ha afirmado que precisamente de ello se derivó el fracaso de preservar la integridad de la monarquía hispánica, ya que los nuevos parámetros políticos «no estaban dispuestos a extender los cambios que en materia de libertades se estaban alcanzando a las posesiones ultramarinas, a riesgo de perder los privilegios históricos» ${ }^{93}$. De hecho, la actitud de las colonias hacia la Metrópoli que había empezado a cambiar a partir de la ocupación napoleónica, lo hizo en mayor medida tras 1810 y 1812, momento en que se incrementaron las aspiraciones de autonomía y comenzaron los procesos independentistas de la mayor parte de ellas.

En los territorios coloniales señalados se pudo observar la misma decepción que se aprecia en Estados Unidos. Casi todas las noticias reproducidas en la prensa de estos últimos provenían de Cuba y Nueva Orleans, con predominio de la primera. Da ejemplo el siguiente fragmento extraído de una carta recibida en Charleston en julio de 1814, bajo el titular «Highly Interesting», $\mathrm{y}$ «Extract of a Letter from a gentleman in Amelia to his friend in this city, dated Amelia July 16».

The most alarming news has reached us concerning the inhabitants of Cuba. Ferdinand the VII having refused to adopt the new Constitution, the people of Spain rose against him. The army is in his favour. When this news reached the Havana, the people were exasperated with the King; every stone in the place were shut up; mobs collected every where, abusing Ferdinand and committing every species of depredation; and plunder was the order of the day! The most alarming scene that could possibly be imagined, was witnessed. How this will terminate God only knows! ${ }^{94}$.

Todos los artículos dedicados a esta cuestión relacionada con la isla de Cuba coinciden en afirmar que una vez se hizo público el desenlace de los acontecimientos en

\footnotetext{
92 Alexandria Gazette (Alexandria, VA), 17 de noviembre de 1812, p. 2.

93 LAFIT, Facundo: «El liberalismo peninsular ante la cuestión americana». Historia Contemporánea, $\mathrm{n}^{\circ}$ 46, 2013, p. 47.

94 Citado en Palladium of Liberty (Morristown, NJ), 4 de agosto de 1814, p. 2; New Jersey Journal (Elizabethtown, NJ), 2 de agosto de 1814, p. 3; Daily National Intelligencer (Washington, DC), 2 de agosto de 1814, p. 3; Delaware Gazette and State Journal (Wilmington, DE), 4 de agosto de 1814, p. 3; Western Citizen (Paris, KY), 13 de agosto de 1814, p. 3; Union (Washington, KY), 13 de agosto de 1814, p. 2; Republican Farmer (Bridgeport, CT), 3 de agosto de 1814, p. 2; Connecticut Courier (Bridgeport, CT), 3 de agosto de 1814, p. 2; Norwich Courier (Norwich, CT), 10 de agosto de 1814, p. 2; American Mercury (Hartford, CT), 9 de agosto de 1814, p. 3; Engine of Liberty and Uniontown Advertiser (Uniontown, MD), 4 de agosto de 1814, p. 3 \& American and Commercial Daily Advertiser (Baltimore, MD), 29 de julio de 1814, p. 3; Weekly Visiter (Kennebunk, ME), 6 de agosto de 1814, p. 2; New Jersey Journal (Trenton, NJ), 2 de agosto de 1814, p. 3; Dartmouth Gazette (Hanover, NH), 10 de agosto de 1814, p. 2; Repertory (Boston, MA), 3 de agosto de 1814, p. 3; Western America (Williambsburg, OH), 6 de agosto de 1814, p. 2; Ohio Republican (Dayton, OH), 15 de agosto de 1814, p. 3; Telescope (Warren, RI), 6 de agosto de 1814, p. 2; Columbian Patriot (Middlebury, VT), 17 de agosto de 1814, p. 2; Enquirer (Richmond, VA), 30 de julio de 1814, p. 3; Farmer's Register (Lansingburg, NY), 9 de agosto de 1814, p. 3; Orange County Gazette (Goshen, NY), 9 de agosto de 1814, p. 2 o New York Gazette (New York, NY), 1 de agosto de 1814, p. 3.
} 
La Habana, el pueblo tuvo que someterse a tal decisión «at the point of the bayonet» que imponían las autoridades ${ }^{95}$. Tras ello «the inhabitants had renounced the Cortes, and attached themselves to Ferdinand 7 th ${ }^{96}$, por lo que ningún altercado tuvo consecuencias mayores ${ }^{97}$. Sin embargo, ese relato de aparente tranquilidad y normalidad ante el cambio político escondía en realidad un proceso previo -aunque breve- en el que «great disturbances had arisen on receipt of the news that Ferdinand had refused to accept to the new constitution of Spain; the city is reported to be in a state of anarchy and confusion -many excesses had been committed, and the inhabitants are highly incensed against the conduct of the king $\rangle^{98}$. Una gran insatisfacción popular, por tanto, al otro lado del Atlántico que se vio sofocada por la aceptación general a los decretos de Fernando VII a que se vio obligada la isla ${ }^{99}$.

Similares son las reflexiones en torno a los territorios de Louisiana ${ }^{100}$, Florida o la región del Missisipi ${ }^{101}$ que todavía se encontraban bajo influencia española; zonas que a su vez estaban directamente vinculadas a los intereses comerciales y territoriales estadounidenses en su frontera sur. Junto a ellas, un caso en el que se comprueba tanto la percepción de la noticia de la derogación de la Constitución de Cádiz como el deseo de emancipación ya existente al menos desde 1810 en ese lado del Atlántico fue el de Méjico. Según informaciones procedentes de allí «that province had declared itself independent (both Royalist and Patriots joining) on receiving information of the rejection by Ferdinand VII, of the constitution formed by the Cortes» ${ }^{102}$. Tras conocer el regreso de Fernando VII y sus decisiones políticas inmediatas, «all Mexico are about to declare themselves independent of that tyrant» que había «ruined his own cause» ${ }^{103}$ :

As soon as it was known at Mexico, that King Ferdinand had rejected the constitution formed by the Cortes, and re-established the Inquisition and other engines of tyranny in Spain, it is started the Royalists united with the Patriots in renouncing all

95 Federal Republican for the Country (Georgetown, DC), 9 de agosto de 1814, p. 1. Esta noticia bajo el titular «By the southern mail. Further particulars relative to Spain», en Federal Republican (Georgetown, DC), 6 de agosto de 1814, p. 2; o, similar, en American Watchman (Wilmington, DE), 13 de agosto de 1814, p. 2.

96 Expresión literal en Weekly Visiter (Kennebunk, ME), 27 de agosto de 1814, p. 3.

97 Según Daily National Intelligencer (Washington, DC), 9 de agosto de 1814, p. 3; Federal Republican (Georgetown, DC), 9 de agosto de 1814, p. 3; American and Commercial Daily Advertiser (Baltimore, MD), 29 de julio de 1814, p. 3 o Weekly Visiter (Kennebunk, ME), 6 de agosto de 1814, p. 2.

98 American Advocate (Hallowell, ME), 13 de agosto de 1814, p. 1.

99 Boston Daily Advertiser (Boston, MA), 4 de agosto de 1814, p. 3.

100 Ejemplo en American Watchman (Wilmington, DE), 22 de octubre de 1814, p. 3.

101 En American and Commercial Daily Advertiser (Baltimore, MD), 17 de octubre de 1814, p. 2 y Baltimore Patriot (Baltimore, MD), 25 de octubre de 1814, p. 2; Weekly Visiter (Kennebunk, ME), 9 de abril de 1814, p. 1; o American Advocate (Hallowell, ME), 13 de diciembre de 1814, p. 4.

102 Western Citizen (Paris, KY), 5 de noviembre de 1814, p. 2. Similar noticia aparecía en el mismo periódico un mes antes el 15 de octubre, en página 2 bajo el titular «Late from New Orleans»; y en Connecticut Herald (New Haven, CT), 25 de octubre de 1814, p. 2; Republican Farmer (Bridgeport, CT), 26 de octubre de 1814, p. 3; Engine of Liberty and Uniontown Advertiser (Uniontown, MD), 27 de octubre de 1814, p. 2; American and Commercial Daily Advertiser (Baltimore, MD), 17 de octubre de 1814, p 3; y Republican Star (Easton, MD), 25 de octubre de 1814, p. 3.

103 Última idea en Connecticut Herald (New Haven, CT), 1 de noviembre de 1814, p. 2. 
connexion with that kingdom, and declared that important, extensive, and fertile American province an independent state ${ }^{104}$.

En este caso no apareció trascripción alguna sobre críticas directas contra el rey o sus decisiones procedentes de los territorios coloniales, todavía sometidos al control español, aunque se desprende por las líneas descritas un descontento evidente y extensible al conjunto de la América Hispana, aunque no se mencionen aquí testimonios de otras colonias.

\section{A modo de conclusión}

El hecho de que en 1814 Fernando VII regresara al trono español imponiendo de nuevo un régimen de tipo absolutista y acabara de raíz con toda la obra liberal gaditana, supuso un duro golpe para el liberalismo español y para las colonias. En 1812 se había sembrado un precedente constitucional y había triunfado un nuevo ideal político, pero habría que esperar hasta la década de los 30 del siglo XIX, tras el fallecimiento del monarca, para ver su consolidación en el establecimiento definitivo del Estado Liberal en el país.

En las páginas de los diarios de Estados Unidos en 1814 se reflejó una profunda admiración por aquel pueblo español que desde 1808 luchaba por defender su independencia, por una España que había logrado vencer a la máquina imperial de Napoleón y poner freno a su política expansiva en el continente. Un país que «for six years, has carried on her bloody war with France. [...] She was, for many years, the only power on the continent that opposed Bonaparte, and with England, was the first to invade his realm. She has found time during her struggle, to infuse the elements of liberty into her political system ${ }^{105}$.

Lo que se criticó en realidad desde esa prensa norteamericana fue la incapacidad de Fernando VII como monarca, «a weak and capricious prince» ${ }^{106}$, así como la cobardía de ese mismo pueblo que finalmente terminó sucumbiendo a los designios absolutistas de aquél. Cambio de actitud que solo se comprende al tener presente el verdadero sentido del levantamiento nacional de 1808. Una revolución que trató de derribar al poder establecido y de crear nuevos órganos de gobierno para sustituirlo; pero en la que no estaba en la mente de sus protagonistas un cambio radical del sistema político, social o económico, sino una clara oposición a la intervención en el país de una política extranjera y la defensa de un rey al que consideraban legítimo ${ }^{107}$. Esta realidad no se ve completamente reflejada en los periódicos estadounidenses, pero es un hecho imprescindible para entender el devenir histórico de los acontecimientos y

104 Northern Post (Salem, NY), 3 de noviembre de 1814, p. 2, donde dice provenir del Mexico Independent.

105 Gazette (Portland, ME), 13 de junio de 1814, p. 3.

106 Así descrito en Chronicle (Harrisburg, PA), 1 de agosto de 1814, p. 3 en un artículo encabezado «From the Democratic Herald. The news from Spain».

107 En estos términos reflexiona el texto de CARANTOÑA ÁLVAREZ, Francisco: «1808, una Revolución incómoda». Spagna Contemporanea, $\mathrm{n}^{\mathrm{0}}$ 42, 2012, pp. 7-26. 
la actitud de la población; independientemente del posicionamiento crítico e ideológico, a favor o en contra, que se mantuviera frente a ellos.

En ese contexto gran parte de la prensa norteamericana reflexionaba con cierta «nostalgia» sobre la situación de Europa en un momento en que todos los gobiernos del continente luchaban por curar «wounds inflicted by the war, to remove grievances, and soften animosities». En esas circunstancias se cuestionaban de una manera crítica "why does not Ferdinand learn a lesson from them? It is said that a strong party has declared itself in favor of King Charles IV. If this be true, it is the strongest evidence of the unpopularity of the present Government; for surely a weaker Monarch [...] never existed» ${ }^{108}$.

El continente vivía un momento de gran inestabilidad y agitación. No se sabía cuál sería la dirección de futuro de los diferentes países a partir de entonces, pero sí quedó claro a ojos de los papeles estadounidenses que «Spain is on an eve of great revolution», que la agonía que había dado comienzo en 1808 no había terminado y todavía estaban por llegar decisiones y cambios políticos que afectaban claramente a las relaciones internacionales según el marco de libertades individuales y políticas que el liberalismo empezaba a reconocer; una serie de sucesos que «may influence in the happiness of the world $»^{109}$. Desde sus páginas, esta prensa se lamentaba con nostalgia: «When the thrones of the French Revolution ended in Despotism, we sighed to see it, and so did every man who loved the cause of liberty in both worlds». Para un país joven, «where it is the very principle of our constitution» $y$ «all power flows from the people, and that all men are born equal and free» ${ }^{110}$, la situación generada en España constituía una amenaza para sus valores morales, sus intereses económicos y sus principios socio-económicos y políticos. Pero sobre todo suponía una gran decepción ante el triste desenlace de un pueblo que, con valentía, coraje y esfuerzo, había conseguido tanto.

How is it possible to restore Spain to her former condition? Will the Spanish nation consent to return to their degraded state? Will they restore to the monarch all his powers, and surrender the sweets of self government? Or is it believed, that a compromise can be made, which shall leave Spain a limited monarchy, with its King, Lords, and Commons?

Can the Bourbon race, on the throne of Spain, defend that country against France? Will not the royal and republican parties in that kingdom quarrel, and one of them again invite France to take part in the contest?

I confess this is what I fear; and I cannot look forward to the future state of the Peninsula without great anxiety ${ }^{111}$.

Las dudas que dejan entrever estas reflexiones terminaron por confirmarse durante las décadas siguientes, tras un nuevo triunfo liberal en 1820 y la segunda restauración absolutista de Fernando VII en 1823, en un proceso lento y gradual hacia un liberalismo tardío que tuvo que esperar al fallecimiento de monarca en 1833 para poder iniciar su despegue definitivo.

108 Del 22 de noviembre de 1814 en Delaware Gazette and State Journal (Wilmington, DE), p. 1; Baltimore Patriot (Baltimore, MD), p. 2 y American and Commercial Daily Advertiser (Baltimore, MD), p. 2.

109 Ambas citas del artículo «Reflections», en Western Citizen (Paris, KY), 6 de agosto de 1814, p. 3.

110 Ideas en American and Commercial Daily Advertiser (Baltimore, MD), 18 de julio de 1814, p. 2.

111 Boston Spectator (Boston, MA), 19 de marzo de 1814, p. 45. 